\title{
Genetic deletion of calcium/calmodulin-dependent protein kinase kinase $\beta$ (CaMKK $\beta$ ) or CaMK IV exacerbates stroke outcomes in ovariectomized (OVXed) female mice
}

Lin Liu', Louise McCullough ${ }^{1,2}$ and Jun Li $i^{*}$

\begin{abstract}
Background: Stroke is the primary cause of long-term disability in the United States. Interestingly, mounting evidence has suggested potential sex differences in the response to stroke treatment in patients as, at least in part, distinct cell death programs may be triggered in females and males following stroke. The $\mathrm{NIH}$ has recognized that females are strikingly under-represented in pre-clinical trials. Calcium/calmodulin-dependent protein kinase kinase (CaMKK) is a major kinase that is activated by elevated intracellular calcium. It has recently been suggested that CaMKK and CaMK IV, a downstream target molecule, are neuroprotective in stroke in males. In this study, we examined stroke outcomes in ovariectomized CaMKK $\beta$ and CaMK IV deficient females. Cell death/survival signaling and inflammatory responses were assessed.

Results: Our results demonstrated that CaMKK $\beta$ or CaMK IV KO exacerbated both ischemic injury and behavioral deficits in female mice. Genetic deletion of CaMKK $\beta$ or CaMK IV increased hemorrhagic transformation after stroke, and this was associated with both increased MMP9 activity and loss of the blood brain barrier (BBB) protein collagen IV. Transcriptional inactivation was observed in mice lacking either CaMKK $\beta$ or CaMK IV, as indicated by reduced levels of phosphorylated CAMP response element-binding protein ( $p$-CREB) and B-cell lymphoma 2 (BCL-2) proteins. Finally, inhibiting this pathway exacerbated the inflammatory response to stroke as CaMKK $\beta$ or CaMK IV KO mice had increased levels of the pro-inflammatory serum cytokines tumor necrosis factor alpha (TNFa) and interleukin 6 (IL-6) after stroke. This suggests that the CaMKK pathway is involved in the immune response to brain injury.
\end{abstract}

Conclusions: Inhibition of CaMKK signaling exacerbated stroke outcome and increased BBB impairment, transcriptional inactivation and inflammatory responses in females after stroke. Therefore, CaMKK signaling may be a potential target for stroke treatment in both males and females.

Keywords: Stroke, CaMKK $\beta$, CaMK IV, Inflammation, Sex differences

\section{Background}

Stroke is the primary cause of long-term disability in the United States [1]. Despite decades of research, the only currently available treatment for stroke is t-PA, which has a very narrow therapeutic window owing to the risk of hemorrhage [2]. The epidemiology of human stroke is sexually dimorphic, and stroke rates are higher in men

\footnotetext{
*Correspondence: junli@uchc.edu

${ }^{1}$ Department of Neuroscience, University of Connecticut Health Center, 263 Farmington Avenue, MC3401, Farmington, CT 06030, USA

Full list of author information is available at the end of the article
}

than in women until advanced ages [3]. This sexual dimorphism is thought to result in part from differences in gonadal hormone levels, particularly estrogen. However, the effect of biological sex on stroke outcome is evident even in clinical populations with only minimal differences in hormone levels, such as neonates and the elderly, which suggests that non-hormonal factors also contribute to outcome [4]. Indeed, experimental stroke studies have demonstrated that distinct cell death programs are triggered in females and males following stroke. For example, poly-ADP-ribose polymerase (PARP) is thought to 
be the dominant pathway for neuronal death in males, whereas in females, caspase-dependent cell death is the dominant pathway after stroke [5]. Based on this mounting evidence supporting potential sex differences in responses to stroke treatment, the criteria developed by the Stroke Therapy Academic Industry Roundtable (STAIR) urge scientists to also study the responses of female animals to experimental stroke [6]. Additionally beginning in October 2014, NIH will require grants applicants to report their plans for the balance of male and female cells and animals in preclinical studies in future applications [7].

Calcium/calmodulin-dependent protein kinase kinase (CaMKK) is a major kinase that is activated by elevated intracellular calcium and is highly abundant in the brain [8]. Upon activation, CaMKK phosphorylates its downstream substrates CaMK I/IV. We have previously shown that inhibiting the CaMKK/CaMK IV pathway exacerbates infarcts and behavioral deficits after cerebral ischemia in male mice, which suggests that this pathway is endogenously protective in stroke [9]. The underlying mechanisms are not fully known, but they may involve increased blood brain barrier (BBB) protection and regulation of transcriptional activation. Interestingly, studies have suggested that the CaMKK pathway has functionally different effects in hippocampal memory formation in male and female mice. In males, CaMKK $\beta$ (one of the two CaMKK isoforms) is required for spatial memory formation, and deleting this gene leads to impairment in long-term potentiation at hippocampal CA1 synapses. However, this deficit was not observed in female mice [10]. This male-specific function of CaMKK in memory formation may be due to gene transcription that only occurs in males [10]. Because transcriptional regulation is thought to be a key mechanism by which CaMKK achieves its neuroprotective role in stroke [9], the role of CaMKK signaling in response to stroke may differ between the sexes. Therefore, we investigated the effects of deleting both CaMKK $\beta$ and one of its two downstream targets, CaMK IV in female mice after stroke.

\section{Methods}

\section{Animals}

All experiments were approved by the Center for Laboratory Animal Care of the University of Connecticut Health Center and were performed in accordance with the National Institutes of Health guidelines for the care and use of laboratory animals. Both the CaMKK $\beta$ knockout (KO) mice and the CaMK IV KO mice were provided by Dr. Anthony Means at Duke University and were backcrossed into the C57BL/ $6 \mathrm{~J}$ background for at least 10 generations. Both $\mathrm{KO}$ strains are normal in size and do not display any gross physical or behavioral abnormalities. F1 heterozygous mattings were used to generate control female mice, which were subsequently backcrossed into the C57BL/6 J background.
Female mice that were age- and weight-matched were used in all experiments (20-24 g, 10-12 weeks of age). All of the mice were OVXed 10 days before undergoing middle cerebral artery occlusion (MCAO) as previously described [11]. We used OVXed animals in this study to control for the levels of gonadal hormones and to reduce the experimental variability that is associated with varying estrogen levels during estrus. This is also a more appropriate model for the "at risk" clinical population because the vast majority of women who experience stroke are postmenopausal [4].

\section{Middle cerebral artery occlusion}

We induced focal transient cerebral ischemia (90 minutes of MCAO) in CaMKK $\beta$ or CaMK IV KO and WT female (control) mice using previously established methods [12]. After 90 minutes of occlusion, the mice were reanesthetized and re-perfused by suture withdrawal. During the ischemic period, their body temperature was controlled at a physiological level using a heating pad with a feedback thermo-control system (FST). The animals were randomized into the stroke and surgical sham cohorts. Mice in the sham group underwent the same procedure except that the MCA was not occluded.

\section{Behavior measurement}

At 72 hours after the onset of stroke, neurological deficits were scored on a four-point scale as previously described, where 0 : no deficit; 1 : forelimb weakness and torso turning to the ipsilateral side when held by the tail; 2: circling to the affected side; 3 : unable to bear weight on the affected side; and 4: no spontaneous locomotor activity or barrel rolling [12].

\section{Hemoglobin assay}

At 72 hours after the onset of stroke, mice were anesthetized with Avertin and perfused transcardially with cold PBS containing heparin $(100 \mathrm{U} / \mathrm{mL})$. The brains were removed, sliced, and initially used for 2,3,5-triphenyl-2 h-tetrazolium chloride (TTC) staining as described below. The brain slices were then dissected to separate the affected and unaffected hemispheres, and quantification of hemorrhagic transformation (HT) was performed using a spectrophotometric hemoglobin assay as previously described [12]. The affected hemispheres were homogenized by sonication in distilled water, followed by centrifugation at $13,000 \mathrm{rpm}$ for 30 minutes. After the supernatant was collected, $200 \mu \mathrm{l}$ of reaction reagent (QuantiChrom Hemoglobin Assay Kit; BioAssay Systems, Hayward, CA, USA) was added to each $50 \mu \mathrm{l}$ of supernatant, and the samples were allowed to stand for 15 minutes before the optical density was measured at $405 \mathrm{~nm}$ using a spectrophotometer (Wallace 1420, PerkinElmer, Waltham, 
MA, USA). Finally, the total hemoglobin concentration was calculated as micrograms per hemisphere [12].

\section{Infarct measurement}

At 72 hours after the onset of stroke, the animals were sacrificed, and the brains were immediately removed and cut into 5 individual $2-\mathrm{mm}$ slices. These brain slices were stained with $1.5 \% 2$, 3, 5-triphenyltetrazolium (TTC) at $37^{\circ} \mathrm{C}$ for 8 minutes. Sigmascan Pro5 software was used to analyze infarct volumes (corrected for edema) after the brain TTC images were digitized, as previously described [13]. Using the same software, edema formation (brain swelling) was calculated. Edema $=100 \%$ * (ipsilateral hemisphere volume - contralateral hemisphere volume)/ contralateral hemisphere volume.

\section{Immunoblotting}

To detect protein levels, mouse brains were harvested 6 hours after the onset of stroke and then homogenized in RIPA lysis buffer (Boston Bioproducts). The concentration of protein in each sample was determined with a BCA assay (Thermo Scientific). The proteins were separated using precast gels $(7.5 \%, 10 \%, 12 \%$ or $4-15 \%$, Bio-Rad) and were then transferred to polyvinylidene difluoride membranes (Bio-Rad). After the transfer and blocking steps, the blots were incubated overnight at $4^{\circ} \mathrm{C}$ with primary antibodies (anti-Collagen IV antibody, 1:500, Abcam; anti-p-CREB antibody, 1:1000, Abcam; or anti-BCL-2 antibody, 1:1000, Cell Signaling Technology) diluted in Tris-buffered saline containing 0.1\% Tween-20 and $4 \%$ bovine serum albumin or $5 \%$ fat free milk. The appropriate secondary antibodies (anti-rabbit IgG, 1:5000, Cell Signaling Technology; or anti-mouse IgG, 1:5000, Vector) were diluted in the same blocking buffer, and an electrochemiluminescence detection kit (Thermo Scientific) was used for signal detection. $\beta$-actin (primary antibody 1:5000; Sigma) was used as a loading control.

\section{Gelatin zymography}

We employed gelatin zymography to assess the activity of matrix metalloproteinase-9 (MMP-9). At 6 hours after stroke, the mice were sacrificed, and their brains were removed and homogenized using RIPA lysis buffer (Cell signaling). Aliquots of the supernatant, each containing $300 \mu \mathrm{g}$ of protein, were subjected to affinity precipitation with gelatin-conjugated sepharose beads (GE, Life Science). The bound material was released from the beads in $50 \mu$ l of elution buffer with $10 \%$ DMSO. Finally, the samples were analyzed with $10 \%$ gelatin zymogram gel (Bio-Rad) [9].

\section{Enzyme-linked immunosorbent assay (ELISA)}

Blood samples were collected transcardially at 72 hours after stroke and were spun at 6000 r.p.m. for 10 minutes at $4^{\circ} \mathrm{C}$. Cytokines were detected by ELISA, performed according to the manufacturer's instructions (eBioscience, US) [12].

\section{Statistical analysis}

All data are presented as the mean \pm SEM except for neurological scores, which are expressed as the median (IQR). One-way ANOVA was used to perform mean comparisons between the experimental groups, and a post-hoc test (Tukey's) was then used for multiple comparisons. Neurological scores were compared with the Mann-Whitney $U$ test. A value of $\mathrm{p}<0.05$ was considered statistically significant. Behavioral and histological assessments were conducted by an investigator who was blinded to the genotype/drug treatment.

\section{Results \\ CaMKK $\beta$ and CaMK IV protein levels did not differ between male and females}

We used Western blots to measure protein levels of CaMKK $\beta$ and CaMK IV at baseline in both males and females. Female ovarectomized mice and male mice displayed equal CaMKK $\beta$ and CaMK IV protein levels in the brain ( $n=6$ for each group) (Figure 1).

\section{CaMKK $\beta$ knockout exacerbated stroke injury}

At 72 hours after stroke onset, CaMKK $\beta$ KO OVXed female mice had significantly larger infarcts in the cortex, the striatum and the total hemisphere compared to WT controls (cortex: KO $66.1 \pm 1.7 \%$ versus WT $55.9 \pm 3.9 \%$, $\mathrm{p}<0.05$; striatum: KO $69.6 \pm 3.1 \%$ versus WT $58.7 \pm$ $4.0 \%, \mathrm{p}<0.05$; total: $\mathrm{KO} 63.1 \pm 2.7 \%$ versus WT $52.6 \pm$ $3.7 \%, \mathrm{p}<0.05, \mathrm{n}=7 \mathrm{WT}$ and $\mathrm{n}=9 \mathrm{KO}$ ) (Figure 2A and B). Exacerbated hemispheric swelling was observed in CaMKK $\beta$ KO females $(10.82 \pm 1.94 \%$ in WT versus $16.74 \pm 1.59 \%$ in CaMKK $\beta$ KO, $n=7 \mathrm{WT}$ and $n=9 \mathrm{KO}$ ) (Additional file 1: Figure S1A). These larger infarcts and exacerbated hemispheric swelling contributed to increased neurological deficit scores, demonstrating a worse functional outcome (Additional file 2: Table S1). Furthermore, knockout of the CaMKK $\beta$ gene increased hemorrhagic transformation as measured by a hemoglobin assay 72 hours after stroke $(85.0 \pm 5.5 \mu \mathrm{g} /$ hemisphere in the WT $(\mathrm{n}=7)$ versus $181.7 \pm 28.7 \mu \mathrm{g} /$ hemisphere in the KO $(\mathrm{n}=9), \mathrm{p}<0.05)$ (Figure $2 \mathrm{C}$ and $\mathrm{D})$. No difference was observed in mortality rates between CaMKK $\beta$ KO and WT mice after stroke.

To confirm that gonadal estrogen was reduced, we weighed the uterus 13 days after the mice underwent ovariectomy. Uterine weights were significantly decreased in both OVXed WT and OVXed CaMKK $\beta$ KO mice $(25.5 \pm 1.9 \mathrm{mg}$ for OVXed WT $(\mathrm{n}=7)$ versus $96.7 \pm$ $20.0 \mathrm{mg}$ for WT sham $(\mathrm{n}=5), \mathrm{p}<0.05 ; 26.2 \pm 1.7 \mathrm{mg}$ for 
A

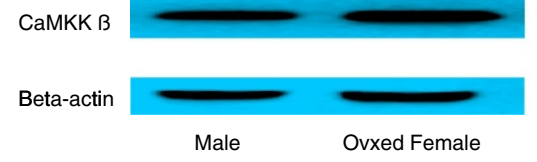

B



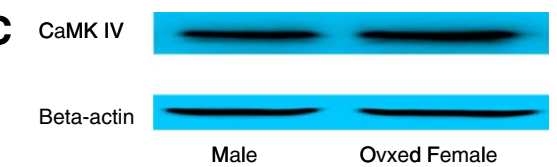

D

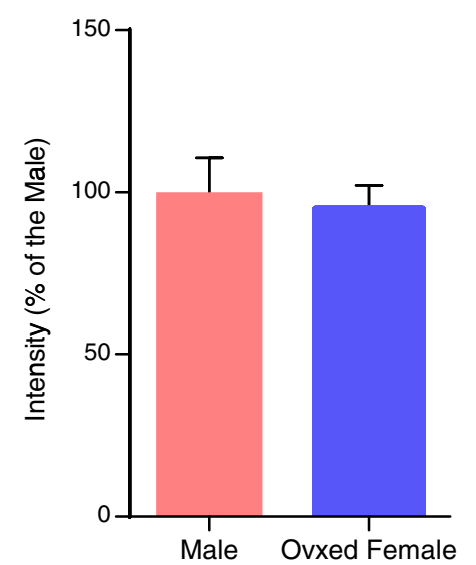

Figure 1 Baseline levels of CaMKK $\beta$ and CaMK IV in males and females. A and C, representative of CaMKK $\beta$ and CaMK IV by Western blot; B and D, quantification of CaMKK $\beta$ and CaMK IV levels, $n=6$ for each group. Data were normalized to corresponding males controls and presented as mean \pm SEM. Ovxed: ovariectomized.

OVXed CaMKK $\beta$ KO ( $\mathrm{n}=9)$ versus $117.5 \pm 16.6 \mathrm{mg}$ for CaMKK $\beta$ KO sham $(\mathrm{n}=5), \mathrm{p}<0.05)$.

\section{CaMK IV knockout worsened stroke outcome}

Knockout of CaMK IV significantly increased the size of infarcts in the cortex, the striatum and total hemisphere at 72 hours after stroke onset (cortex: $66.1 \pm 3.1 \%$ in the $\mathrm{KO}$ versus $56.5 \pm 2.6 \%$ in the WT, $\mathrm{p}<0.05$, striatum: $72.6 \pm 3.4 \%$ in the $\mathrm{KO}$ versus $60.0 \pm 2.5 \%$ in the WT, $\mathrm{p}<0.05$, total: $61.6 \pm 3.0 \%$ in the $\mathrm{KO}$ versus $46.1 \pm$ $4.0 \%$ in the WT $\mathrm{p}<0.05, \mathrm{n}=8$ for each group) (Figure $2 \mathrm{E}$ and F). Exacerbated hemispheric swelling was observed in CaMK IV KO females $(11.24 \pm 1.68 \%$ in WT versus $17.29 \pm 2.41 \%$ in CaMK IV KO, $\mathrm{n}=8$ for each group) (Additional file 1: Figure S1B). Additionally, CaMK IV $\mathrm{KO}$ mice performed worse on neurological deficit tests (Additional file 2: Table S1). Furthermore, the loss of CaMK IV significantly increased hemorrhagic transformation as evaluated by a hemoglobin assay 72 hours after stroke $(82.5 \pm 8.6 \mu \mathrm{g} /$ hemisphere in the WT versus $207.3 \pm 33.2 \mu \mathrm{g} / \mathrm{hemisphere} \mathrm{in} \mathrm{the} \mathrm{CaMK} \mathrm{IV} \mathrm{KO,} \mathrm{p}<0.05$, $\mathrm{n}=8$ for each group) (Figure $2 \mathrm{G}$ and $\mathrm{H}$ ). This result was similar to our previous observation in CaMKK $\beta$ KO mice. No difference in mortality rate was observed between CaMK IV KO and WT mice after stroke.

Similarly, uterine weights of OVXed CaMK IV KO mice and the corresponding OVXed WT controls were also significantly decreased compared to mice with intact ovaries $(22.8 \pm 1.9 \mathrm{mg}$ for OVXed WT $(\mathrm{n}=8)$ versus $102.4 \pm 23.2 \mathrm{mg}$ for WT sham $(\mathrm{n}=5), \mathrm{p}<0.05 ; 23.7 \pm 1.9 \mathrm{mg}$ for OVXed CaMK IV KO $(\mathrm{n}=8)$ versus $106.3 \pm 16.8 \mathrm{mg}$ for CaMK IV KO sham $(\mathrm{n}=5), \mathrm{p}<0.05)$.

Loss of CaMKK $\beta$ or CaMK IV increased gelatinase activity and reduced collagen IV levels 6 hours after stroke

To explore the mechanisms underlying the increased HT in both CaMKK $\beta$ KO and CaMK IV KO mice, we examined the activity of matrix metalloproteinases (MMPs) after stroke onset. Loss of CaMKK $\beta$ resulted in significantly upregulated MMP-9 levels 6 hours after stroke (Figure $3 \mathrm{~A}$ and $\mathrm{C}, \mathrm{n}=3$ per stroke group, $\mathrm{p}<0.05$ ). Similar results were obtained with CaMK IV KO mice (Figure 3B and D, $\mathrm{n}=3$ per stroke group, $\mathrm{p}<0.05$ ). Furthermore, we used Western blotting of samples from 6 hours after stroke onset to quantify the levels of collagen IV protein, which is an important component of the BBB and a substrate of MMPs. Collagen IV levels were decreased in both $\mathrm{KO}$ strains after stroke (Figure 3E-H, $\mathrm{n}=3$ for the sham group, $\mathrm{n}=4$ per stroke group). Additionally, in the sham groups, we observed no difference between the $\mathrm{KO}$ animals and their corresponding WT controls, implying that the loss of either CaMKK $\beta$ or CaMK IV did not affect the baseline levels of BBB integrity (Figure 3E-H, $\mathrm{n}=3$ for the sham group, $\mathrm{n}=4$ for each stroke group).

\section{Genetic deletion of CaMKK $\beta$ or CaMK IV reduced $p$-CREB} and BCL-2 levels 6 hours after stroke

Next, we examined the levels of p-CREB, which is a neuroprotective transcription factor that is activated by CaMK IV [14]. At 6 hours after stroke, p-CREB levels were 


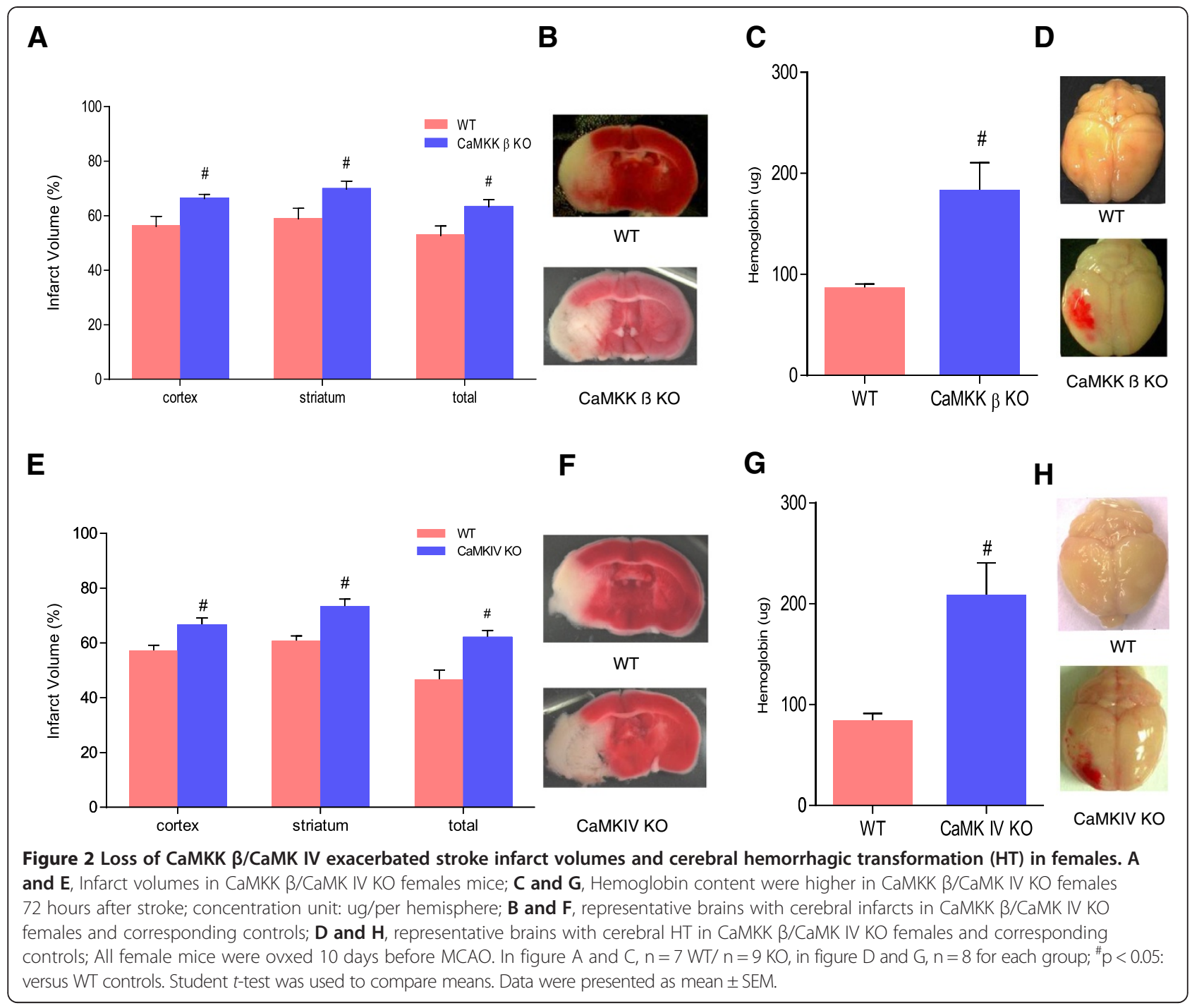

significantly reduced in CaMKK $\beta$ KO mice when compared to their corresponding WT controls (Figure 4A and $\mathrm{C} ; \mathrm{n}=2$ for the sham group; $\mathrm{n}=3$ for the stroke group). A similar reduction in p-CREB levels was observed in CaMK IV KO mice 6 hours after stroke (Figure 4B and D; $\mathrm{n}=2$ for the sham group; $\mathrm{n}=3$ for the stroke group). BCL-2, which is a downstream target of CREB, has previously been shown to be beneficial in stroke. We therefore studied BCL-2 levels in both CaMKK $\beta$ KO and CaMK IV KO mice. Consistent with the p-CREB data, we observed that at 6 hours after stroke, BCL-2 expression was significantly reduced in both $\mathrm{KO}$ strains when compared to their corresponding WT controls (Figure 5A-D, $\mathrm{n}=2$ for the WT group; $\mathrm{n}=3$ for each $\mathrm{KO}$ group).

\section{Loss of CaMKK $\beta$ or CaMK IV aggravated the inflammatory response after stroke}

CaMKK signaling has been implicated in immune regulation, which plays a pivotal role in determining stroke outcome. Therefore, we measured serum levels of IL-6 and TNF $\alpha$ in CaMKK $\beta$ and CaMK IV KO mice 72 hours after stroke. The loss of either CaMKK $\beta$ or CaMK IV aggravated the inflammatory response; this was indicated by increased levels of the pro-inflammatory cytokines TNF $\alpha$ (Figure 6A and B) and IL-6 (Figure 6C and D) $(n=3$ for each group).

\section{Discussion}

In this study, we utilized OVXed female mice, a translationally relevant model system, to explore the role of the CaMKK/CaMK IV pathway in stroke. First, we demonstrated that levels of CaMKK $\beta$ and CaMK IV proteins in the female brain were equivalent to that in the males. Second, we found that the genetic deletion of CaMKK $\beta$ or CaMK IV aggravated both infarcts and behavioral deficits. Third, both CaMKK $\beta$ and CaMK IV KO mice exhibited increased hemorrhagic transformation after stroke, and this was associated with both increased 


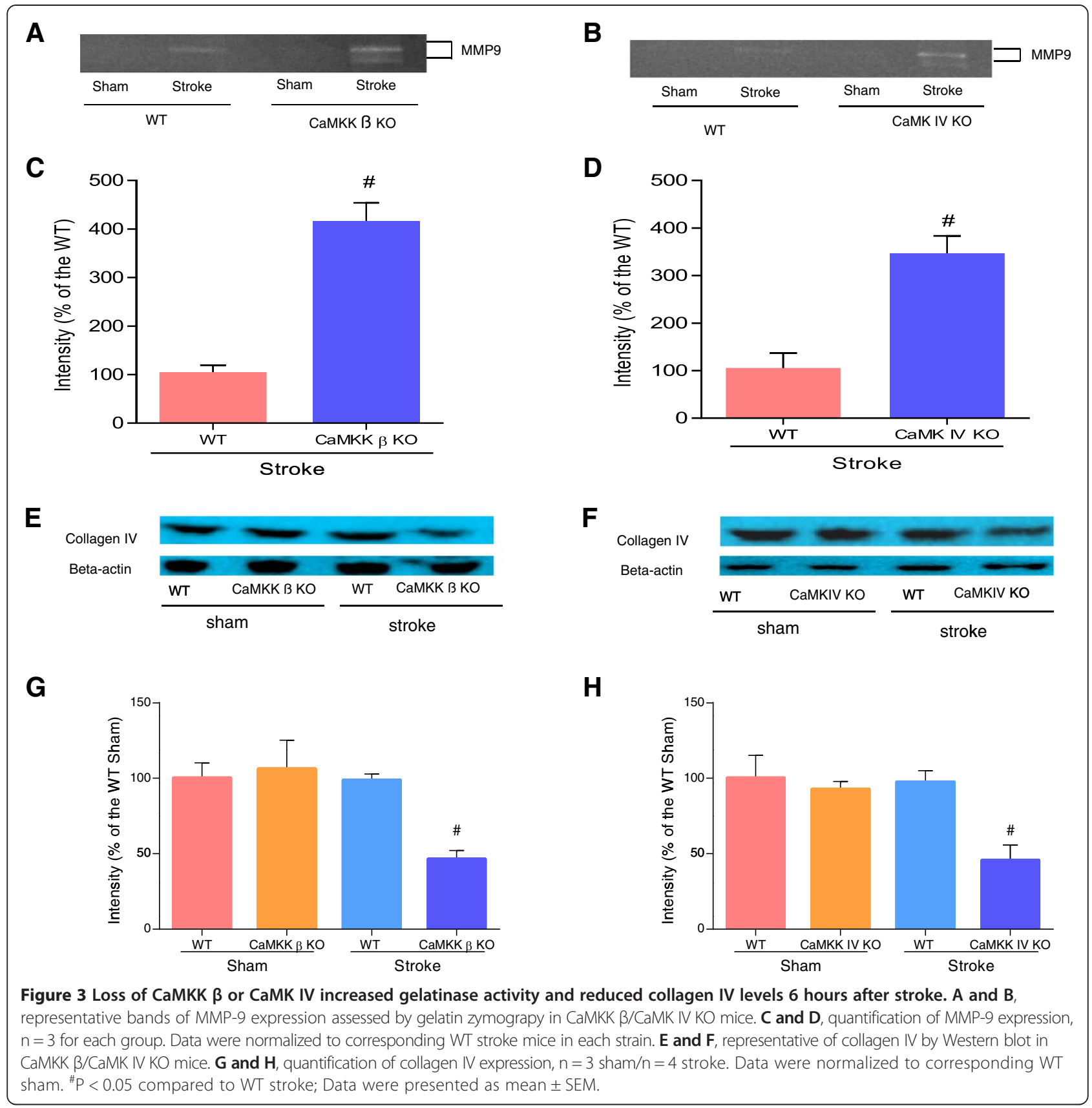

MMP9 activity and the loss of the BBB protein collagen IV. Fourth, transcriptional inactivation was observed in mice lacking either CaMKK $\beta$ or CaMK IV, as indicated by the reduced levels of p-CREB and BCL-2. Finally, we demonstrated that the levels of the proinflammatory serum cytokines TNF $\alpha$ and IL- 6 increased after stroke in both CaMKK $\beta$ and CaMK IV KO mice, indicating that inhibiting this pathway exacerbates the inflammatory response.

Our data demonstrate that CaMKK signaling may be an endogenous protective mechanism in females even though calcium signaling has traditionally been thought to play a detrimental role in stroke outcome. Dramatic intracellular calcium elevation results from the hyperactivation of glutamate receptors and concurrent calcium release from intracellular storage [15]. Elevated intracellular calcium subsequently activates calciumdependent enzymes, including DNases, proteases, and lipases, resulting in cell death under ischemic conditions [16]. However, clinical trials testing calcium blockers have failed to provide neuroprotection in patients after cerebral ischemia [17]. Previous studies have increasingly supported the idea that enhancing calcium also plays a neuroprotective role by triggering endogenous protective 


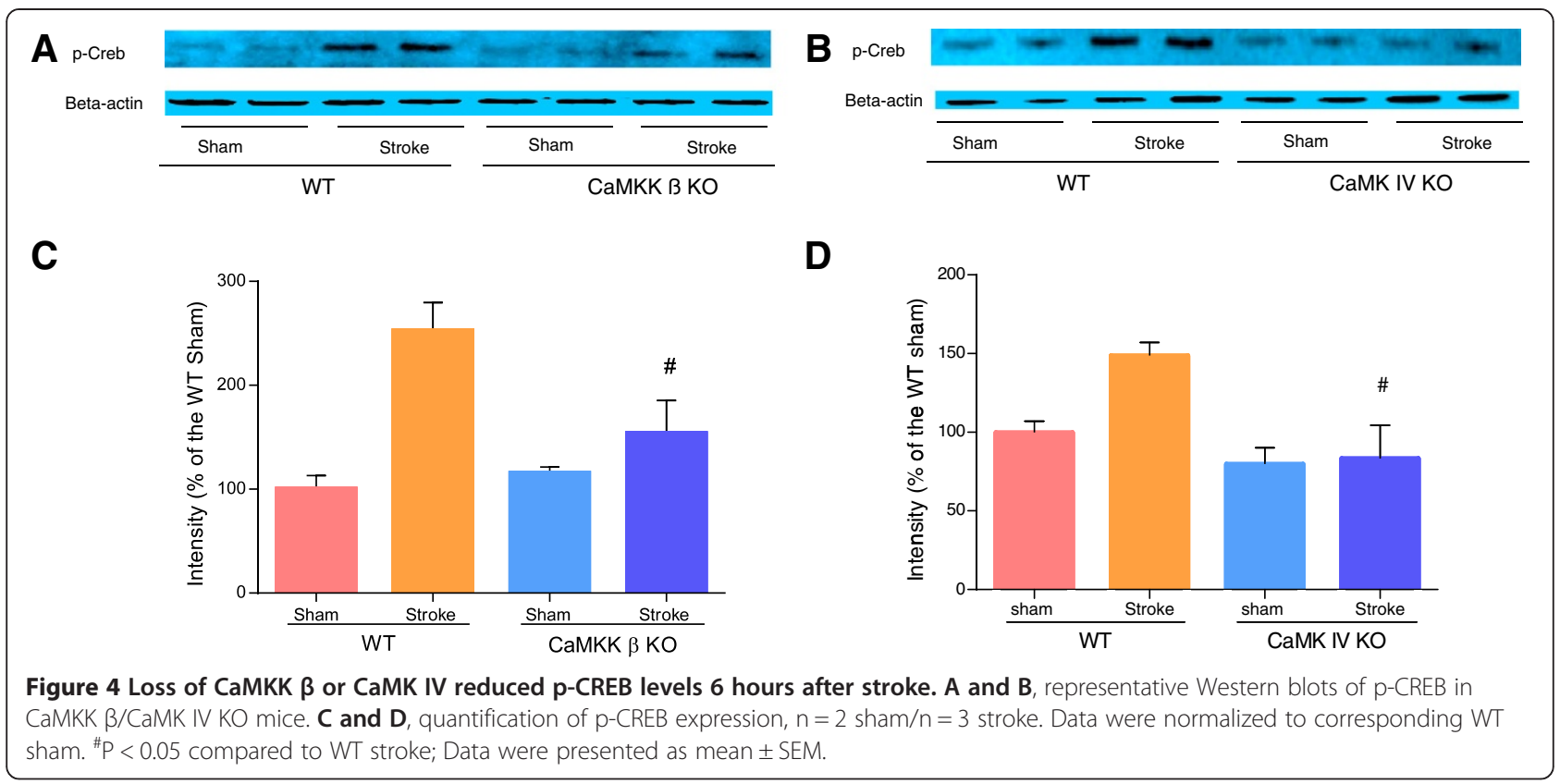

pathways, which may explain the failure of these clinical trials. CaMKK is a major kinase that is activated by intracellular calcium, and it targets multiple critical pathways for neuronal death/survival. Here, we have demonstrated that eliminating CaMKK $\beta$ or CaMK IV exacerbates infarcts, edema formation and behavioral deficits. We have previously demonstrated the effects of CaMKK in maintaining the integrity of the $\mathrm{BBB}$ after stroke, which is particularly important because $\mathrm{BBB}$ disruption is one of the major contributing factors to edema formation and hemorrhagic transformation in cerebral ischemia $[9,18]$. The inhibitory effect of CaMKK on peripheral neutrophil activation and egress has been suggested as a possible mechanism for BBB protection [9]. Additionally, we provide evidence that CaMKK signaling may function to augment the transcription of neuroprotective genes such as BCL-2. Previous studies have suggested that deleting either CaMKK $\beta$ or CaMK IV in males exacerbates the nuclear accumulation of histone deacetylase 4 (HDAC4) [9], which may in turn

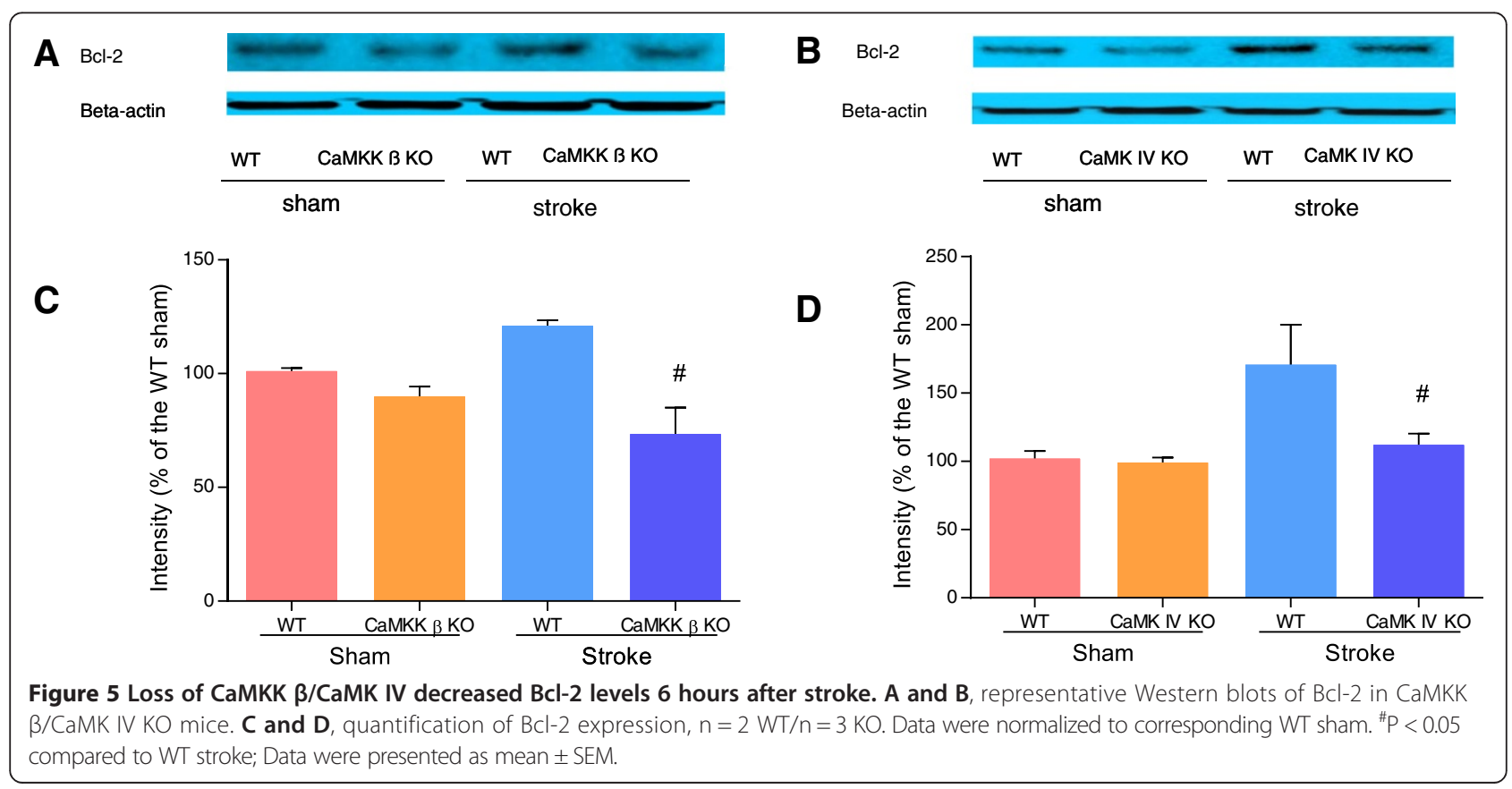




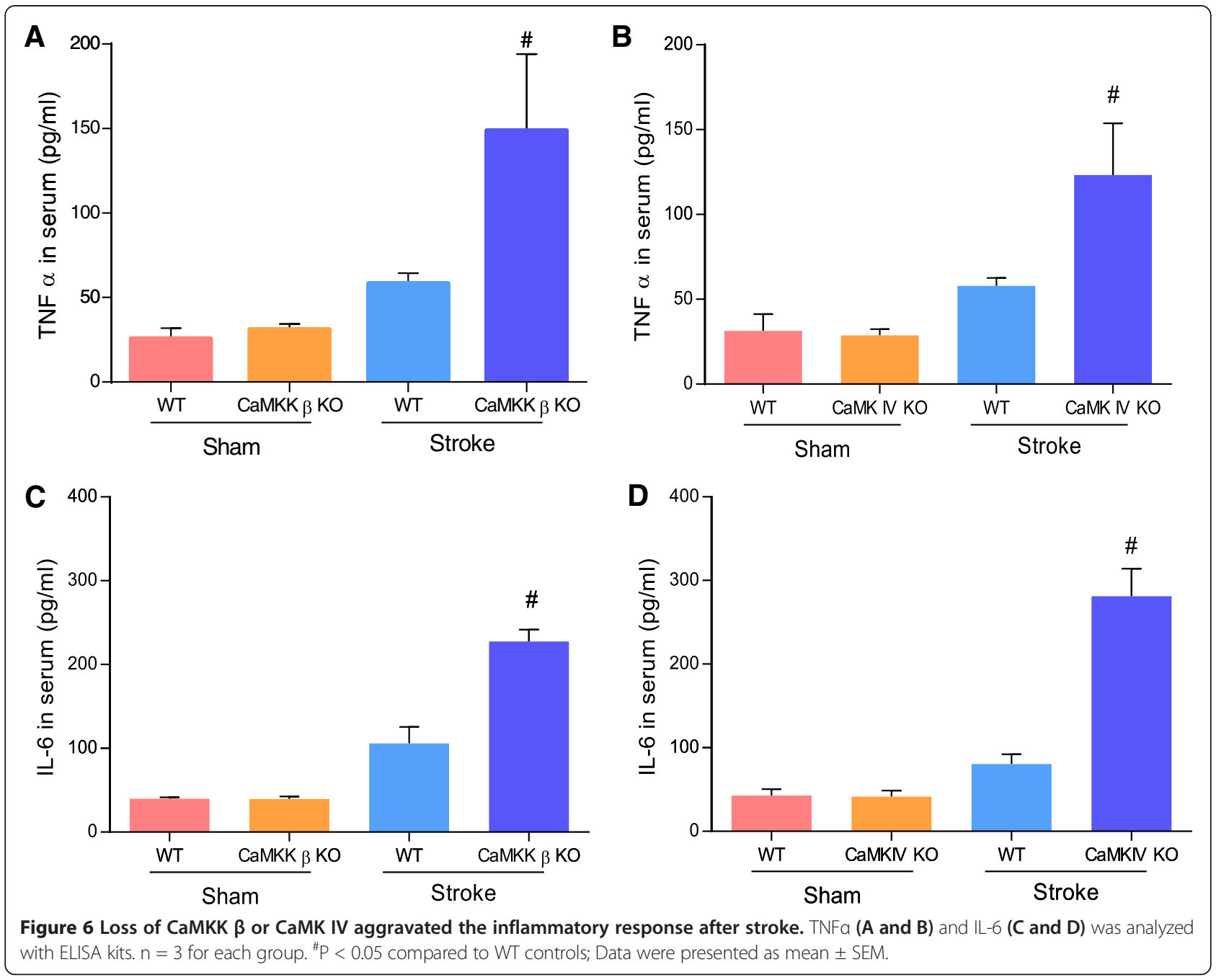

reduce the levels of the neuronal survival factors p-CREB and BCL-2 [19-21]. However, a direct link between CaMKK signaling and the regulation of HDAC4 translocation in females after stroke has not yet been established. Of note, endothelial cell, an important component of the neurovascular unit, may contribute to stroke outcome, particularly changes of $\mathrm{BBB}$ integrities after stroke. Interestingly, endothelial cells also express CaMKK [9]. Based on our data demonstrating a worse $\mathrm{BBB}$ in the $\mathrm{KO}$ after stroke, we speculate that inhibition of CaMKK signaling may enhance endothelia cell death and may contribute to greater HT observed in the KO mice. However, the role of CaMKK in endothelium under ischemic stress is still unclear and remains to be investigated.

Our data highlighted the importance of CaMKK signaling in protecting $\mathrm{BBB}$ in stroke. t-PA is currently the only FDA approved drug for stroke treatment but has a very narrow therapeutic window due to its risk of inducing HT. It would be very interesting in the future to study if targeting CaMKK signaling help reduce the risk of $\mathrm{HT}$ and extend the therapeutic window of t-PA. Indeed, bigger infarcts in $\mathrm{KO}$ mice may lead to worse $\mathrm{BBB}$ disruption and subsequently higher levels of hemoglobin in stroke brain. We therefore did a correlation analysis (data not shown) to see if higher amounts of hemoglobin is correlated to higher infarct volumes in both $\mathrm{KO}$ mice. No significant correlation was observed although there was a trend toward statistical significance in the CaMKK $\beta$ KO mice $(p=0.11)$. We chose 6 hour time point to examine the changes of MMP activity and BBB protein levels et al., as at this time point, stroke infarct is not yet mature. Therefore changes in molecule signaling are more likely to be mechanistic than only a correlation with infarct size. Additionally, our previous published data suggested at this time point, there were significance changes of molecule signaling in males in CaMKK KO and CaMK IV KO mice after stroke [9]. In the current study we would like to see if the similar changes were occurring in females. We will examine the temporal profile of these molecule changes after stroke in our future studies. 
In addition to its effects on BBB integrity and transcriptional activation, our data demonstrate that CaMKK signaling may induce neuroprotection through a reduction in post-stroke inflammation. The inflammatory response is increasingly recognized as a key contributor to stroke damage and is an attractive target for neuroprotective therapies because it takes place in a delayed manner. The CaMKK pathway regulates the activity of several transcription factors such as CREB, activator protein-1 (AP-1), myocyte enhancer factor-2 (MEF2), and members of the retinoid orphan receptor (ROR) family, which are all proteins that play pivotal roles in the immune response and inflammation by regulating processes such as T-cell development and cytokine secretion [14,22-25]. For example, CaMK IV stimulates ROR $\alpha$-dependent transcriptional activation, which is responsive to changes in intracellular calcium in $\mathrm{HaCaT}$ cells [22]. ROR $\alpha$ has been suggested to function as a negative regulator of cytokines such as IL-6 and TNF $\alpha$ because the genetic deletion of ROR $\alpha$ greatly enhances the induction of these cytokines in mast cells and macrophages after LPS treatment [26,27]. Consistent with these earlier in vitro studies, we found that knockout of CaMKK $\beta$ or CaMK IV increased TNF $\alpha$ and IL- 6 production in mice after stroke. Therefore, the exacerbation of the inflammatory response seen in these $\mathrm{KO}$ mice may contribute to their worsened outcome after stroke. However, the mechanism by which CaMKK signaling reduces the inflammatory response after stroke and whether ROR $\alpha$ is involved in this process remains unclear. Of note, in the current study we only measured the inflammatory markers in peripheral blood after stroke. Although it is well established that peripheral inflammatory factors are able to infiltrate into the brain and exacerbate stroke outcome, our future studies will directly focus on the inflammatory response in the brain.

We used ovariectomized $\mathrm{KO}$ and WT control mice in this study primarily because these animals may be a more appropriate model for women at the highest risk for stroke, the majority of whom are postmenopausal [4]. Additionally, performing ovarietomy in female mice allows us to control levels of gonadal hormones therefore to reduce experimental variability that is associated with varying estrogen levels during estrus. We confirmed the loss of gonadal production of estrogen by showing a dramatic reduction in uterine weights after OVX. However, the direct effects of estrogen on the CaMKK signaling pathway in stroke remain unknown because we did not directly study stroke outcome in ovary-intact or estrogen-supplemented OVXed KO females.

Although CaMKK $\beta$ has been shown to affect hippocampal memory formation in a sexually dimorphic manner [28], we found no overall difference between the sexes in the role of this pathway in response to stroke.
In fact, deleting CaMKK or CaMK IV at the genetic level exacerbated the stroke outcome in both males [9] and females in a nonspecific manner. This suggests that CaMKK signaling plays a protective role for both male and female stroke victims. Discrepancies between this result and an earlier report that found sex-specific differences may result from differences in models or particularly in the brain regions targeted in each case. For example, the hippocampus is not directly affected by MCA occlusion, although it may be affected through secondary ischemia caused by stroke-induced brain edema formation. We did not specifically assess memory formation after stroke in females because the primary focus of our study was to identify whether the CaMKK pathway can exert neuroprotective properties in female animals; otherwise, targeting this pathway in females might fail to reduce injury.

\section{Conclusions}

In summary, we have demonstrated that genetically inhibiting the CaMKK pathway is detrimental in the response of female mice to cerebral ischemia. Therefore, CaMKK signaling may play a neuroprotective role in both male [9] and female stroke victims. This result suggests that the CaMKK pathway may be a potential target for stroke therapy.

\section{Additional files}

Additional file 1: Figure S1. Effect of CaMKK $\beta$ and CaMK IV on hemispheric swelling after stroke. Swelling (\%) = (Ipsilateral hemisphere- Contralateral hemisphere)/Contralateral hemisphere *100\%. A, $n=7 \mathrm{WT} / \mathrm{n}=9 \mathrm{KO}, \mathrm{B}, \mathrm{n}=8$ for each group; $\mathrm{p}<<0.05$ : versus WT controls. Data were showed as mean \pm SEM.

Additional file 2: Table S1. Ovxed CaMKK $\beta /$ CaMK IV KO mice had increased neurological deficit scores after stroke.

\section{Abbreviations}

CaMKK: Calcium/calmodulin-dependent protein kinase kinase; BBB: Blood brain barrier; $\mathrm{p}$-CREB: Phosphorylated CAMP response element-binding protein; BCL-2: B-cell lymphoma 2; TNFa: Tumor necrosis factor alpha; IL-6: Interleukin 6; PARP: Poly-ADP-ribose polymerase; STAIR: Stroke Therapy Academic Industry Roundtable; AP-1: Activator protein-1; MEF2: Myocyte enhancer factor-2; ROR: Retinoid orphan receptor.

\section{Competing interests}

The authors declare that they have no competing interests.

\section{Authors' contributions}

$J L$ designed the experiments. $L L$ carried out the experiments. $J L$ and $L L$ analyzed and interpreted the data. The manuscript was written by $J L$ and $L L$. LM helped the interpretation and analysis of the data. All authors read and approved the final manuscript.

\section{Acknowledgements}

This work was supported by NIH grants R01 NS078446 (J. L.) and R21 NS079137 (J.L.).

\section{Author details}

'Department of Neuroscience, University of Connecticut Health Center 263 Farmington Avenue, MC3401, Farmington, CT 06030, USA. 
${ }^{2}$ Department of Neurology, University of Connecticut Health Center, Farmington, CT, USA.

Received: 25 June 2014 Accepted: 8 October 2014

Published online: 21 October 2014

\section{References}

1. Go AS, Mozaffarian D, Roger VL, Benjamin EJ, Berry JD, Blaha MJ, Dai S, Ford ES, Fox CS, Franco S, Fullerton HJ, Gillespie C, Hailpern SM, Heit JA, Howard VJ, Huffman MD, Judd SE, Kissela BM, Kittner SJ, Lackland DT, Lichtman JH, Lisabeth LD, Mackey RH, Magid DJ, Marcus GM, Marelli A, Matchar DB, McGuire DK, Mohler ER III, Moy CS, et al: Heart disease and stroke statistics-2014 update: a report from the American Heart Association. Circulation 2014, 129:e28-e292.

2. Del Zoppo GJ, Saver JL, Jauch EC, Adams HP Jr, American Heart Association Stroke Council: Expansion of the time window for treatment of acute ischemic stroke with intravenous tissue plasminogen activator a science advisory from the American Heart Association/American Stroke Association. Stroke 2009, 40:2945-2948.

3. Manwani B, McCullough LD: Sexual dimorphism in ischemic stroke: lessons from the laboratory. Womens Health (Lond Engl) 2011, 7:319-339.

4. Li J, Benashski SE, Siegel C, Liu F, McCullough LD: Adenosine monophosphate activated protein kinase inhibition is protective in both sexes after experimental stroke. Neurosci Lett 2010, 482:62-65.

5. Yuan M, Siegel C, Zeng Z, Li J, Liu F, McCullough LD: Sex differences in the response to activation of the poly (ADP-ribose) polymerase pathway after experimental stroke. Exp Neurol 2009, 217:210-218.

6. Fisher M, Feuerstein G, Howells DW, Hurn PD, Kent TA, Savitz SI, Lo EH STAIR Group: Update of the stroke therapy academic industry roundtable preclinical recommendations. Stroke 2009, 40:2244-2250.

7. Clayton JA, Collins FS: Policy: NIH to balance sex in cell and animal studies. Nature 2014, 509:282-283.

8. Soderling TR: The Ca-calmodulin-dependent protein kinase cascade. Trends Biochem Sci 1999, 24:232-236.

9. McCullough LD, Tarabishy S, Liu L, Benashski S, Xu Y, Ribar T, Means A, Li J. Inhibition of calcium/calmodulin-dependent protein kinase kinase $\beta$ and calcium/calmodulin-dependent protein kinase IV is detrimental in cerebral ischemia. Stroke 2013, 44:2559-2566.

10. Mizuno K, Antunes-Martins A, Ris L, Peters M, Godaux E, Giese KP: Calcium/ calmodulin kinase kinase beta has a male-specific role in memory formation. Neuroscience 2007, 145:393-402.

11. Li J, Siegel M, Yuan M, Zeng Z, Finnucan L, Persky R, Hurn PD, McCullough LD: Estrogen enhances neurogenesis and behavioral recovery after stroke. $J$ Cereb Blood Flow Metab 2011, 31:413-425.

12. Liu L, Doran S, Xu Y, Manwani B, Ritzel R, Benashski S, McCullough L, Li J: Inhibition of mitogen-activated protein kinase phosphatase-1 (MKP-1) increases experimental stroke injury. Exp Neurol 2014, in press.

13. Li J, Benashski SE, Venna VR, McCullough LD: Effects of metformin in experimental stroke. Stroke 2010, 41:2645-2652.

14. Sée V, Boutillier AL, Bito H, Loeffler JP: Calcium/calmodulin-dependent protein kinase type IV (CaMKIV) inhibits apoptosis induced by potassium deprivation in cerebellar granule neurons. FASEB J 2001, 15:134-144.

15. Besancon E, Guo S, Lok J, Tymianski M, Lo EH: Beyond NMDA and AMPA glutamate receptors: emerging mechanisms for ionic imbalance and cell death instroke. Trends Pharmacol Sci 2008, 29:268-275.

16. Nicotera P: Molecular switches deciding the death of injured neurons. Toxicol Sci 2003, 74:4-9.

17. Cheng YD, Al-Khoury L, Zivin JA: Neuroprotection for ischemic stroke: two decades of success and failure. NeuroRx 2004, 1:36-45.

18. Latour LL, Kang DW, Ezzeddine MA, Chalela JA, Warach S: Early blood-brain barrier disruption in human focal brain ischemia. Ann Neurol 2004, 56:468-677.

19. Li J, Chen J, Ricupero CL, Hart RP, Schwartz MS, Kusnecov A, Herrup K: Nuclear accumulation of HDAC4 in ATM deficiency promotes neurodegeneration in ataxia telangiectasia. Nat Med 2012, 18:783-790.

20. Paroni G, Fontanini A, Cernotta N, Foti C, Gupta MP, Yang XJ, Fasino D, Brancolini C: Dephosphorylation and caspase processing generate distinct nuclear pools of histone deacetylase 4. Mol Cell Biol 2007, 27:6718-6732

21. Kitagawa K: CREB and cAMP response element-mediated gene expression in the ischemic brain. FEBS J 2007, 274:3210-3217.
22. Kane CD, Means AR: Activation of orphan receptor-mediated transcription by $\mathrm{Ca}(2+) /$ calmodulin-dependent protein kinase IV. EMBO J 2000, 19:691-701.

23. Ermisch M, Firla B, Steinhilber D: Protein kinase A activates and phosphorylates RORa4 in vitro and takes part in RORa activation by CaMK-IV. Biochem Biophys Res Commun 2011, 408:442-446.

24. Racioppi L, Means AR: Calcium/calmodulin-dependent kinase IV in immune and inflammatory responses: novel routes for an ancient traveller. Trends Immunol 2008, 29:600-607.

25. Anderson KA, Ribar TJ, Lin F, Noeldner PK, Green MF, Muehlbauer MJ, Witters LA, Kemp BE, Means AR: Hypothalamic CaMKK2 contributes to the regulation of energy balance. Cell Metab 2008, 7:377-388.

26. Dzhagalov I, Giguère V, He YW: Lymphocyte development and function in the absence of retinoic acid-related orphan receptor alpha. J Immunol 2004, 173:2952-2959.

27. Kopmels B, Mariani J, Delhaye-Bouchaud N, Audibert F, Fradelizi D, Wollman EE: Evidence for a hyperexcitability state of staggerer mutant mice macrophages. J Neurochem 1992, 58:192-199.

28. Mizuno K, Ris L, Sánchez-Capelo A, Godaux E, Giese KP: Ca2+/calmodulin kinase kinase alpha is dispensable for brain development but is required for distinct memories in male, though not in female, mice. Mol Cell Biol 2006, 26:9094-9104

doi:10.1186/s12868-014-0118-2

Cite this article as: Liu et al: Genetic deletion of calcium/calmodulindependent protein kinase kinase $\beta$ (CaMKK $\beta$ ) or CaMK IV exacerbates stroke outcomes in ovariectomized (OVXed) female mice. BMC Neuroscience 2014 15:118.

\section{Submit your next manuscript to BioMed Central and take full advantage of:}

- Convenient online submission

- Thorough peer review

- No space constraints or color figure charges

- Immediate publication on acceptance

- Inclusion in PubMed, CAS, Scopus and Google Scholar

- Research which is freely available for redistribution
C Biomed Central 